\title{
Extragalactic origin confirmed
}

Science 357, 1266-1270 (2017)
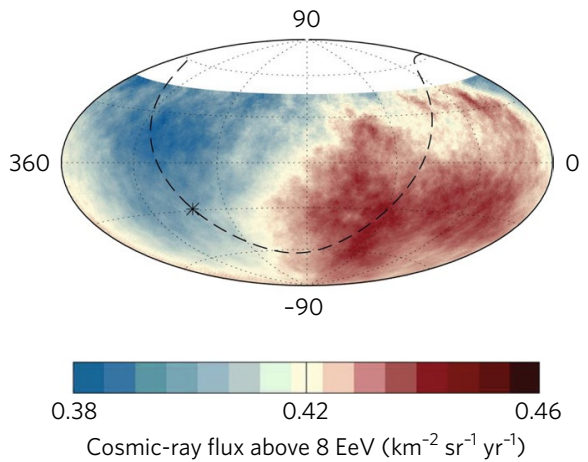

Credit: AAAS

Cosmic rays - fast-moving, high-energy nuclei - pervade the Universe. We know that the lower-energy variety that we detect on Earth is funnelled by the solar wind. However, higher-energy cosmic rays have an isotropic distribution due to scattering that makes it difficult to identify their source, although they are likely to be generated by high-energy phenomena like supernova explosions and jets from active galactic nuclei. By looking at the ultrahigh-energy end of the cosmic ray spectrum (on the order of exa-electron volts and higher, where cosmic rays are not scattered by solarscale magnetic fields), the Pierre Auger Collaboration detected an anisotropy in their arrival directions that indicates an extragalactic origin.
Ultrahigh-energy cosmic rays are rare: typically one cosmic ray with an energy $>10 \mathrm{EeV}$ hits each square kilometre of the Earth's surface per year. The Pierre Auger Observatory in Argentina detects cosmic rays using two combined techniques: telescopes to detect fluorescence from cosmic-ray-generated air showers, and a network of 12-tonne containers of ultrapure water, spread over an area of 3,000 square kilometres. Photomultiplier detectors in the containers observe the faint Cherenkov radiation generated when cosmic-raygenerated muons encounter water molecules. By reconstructing the cone of emission of the muon (analogous to an aircraft's sonic boom) an incident direction can be derived. By analysing 32,187 cosmic rays detected over 12.75 years, a map of the sky was produced (pictured), showing evidence of an enhancement (5.2 $\sigma$ significance) in a region away from the Galactic Centre (marked with an asterisk; the dashed line indicates the Galactic plane). The distance of this hotspot from the Galactic Centre $\left(\sim 125^{\circ}\right)$ points towards an extragalactic origin of ultrahigh-energy cosmic rays, reinforcing previous (less conclusive) results from the Collaboration at lower energies.

\section{Paul Woods}

Published online: 16 October 2017 https://doi.org/10.1038/s41550-017-0304-0 